PROCEEDINGS OF THE

AMERICAN MATHEMATICAL SOCIETY

Volume 130, Number 9, Pages 2761-2770

S 0002-9939(02)06340-2

Article electronically published on March 13, 2002

\title{
ASYMPTOTIC BEHAVIOR OF ROOTS OF RANDOM POLYNOMIAL EQUATIONS
}

\author{
EFRAIM SHMERLING AND KENNETH J. HOCHBERG
}

(Communicated by Claudia M. Neuhauser)

\begin{abstract}
We derive several new results on the asymptotic behavior of the roots of random polynomial equations, including conditions under which the distributions of the zeros of certain random polynomials tend to the uniform distribution on the circumference of a circle centered at the origin. We also derive a probabilistic analog of the Cauchy-Hadamand theorem that enables us to obtain the radius of convergence of a random power series.
\end{abstract}

\section{$\S 1$. InTRODUCTION AND STATEMENT OF MAIN RESULTS}

Convergence and limit theorems are of fundamental importance in the theory of random polynomials, and they have been the subject of active research for the past forty years. Most of the early results in this field are summarized in the monograph by Bharucha-Reid and Sambandham [5, pp. 173-195]. The survey paper by Edelman and Kostlan 7] contains more recent developments in this area, and applications of these ideas to problems in quantum chaotic dynamics are discussed in the paper by Bogomolny et al [6].

In this article, we prove some theorems on the asymptotic behavior of the roots of random polynomial equations whose order tends to infinity. We also derive formulas which enable us to find the radius of convergence of a random power series.

We consider a sequence of random polynomials $\left\{F_{n}(z, \omega)=\sum_{k=0}^{n} a_{k}(\omega) z^{k}\right\}_{n=1}^{\infty}$ whose coefficients form a sequence of independent real- or complex-valued random variables. Clearly, the complex-valued coefficients $a_{k}(\omega)$ are of the form

$$
a_{k}(\omega)=\alpha_{k}(\omega)+i \beta_{k}(\omega)
$$

with means $\mu_{k}$ and variances $\sigma_{k}^{2}$ given by

$$
\begin{gathered}
\mu=\mu_{\alpha_{k}}+i \mu_{\beta_{k}}, \\
\sigma_{k}^{2}=\sigma_{\alpha_{k}}^{2}+\sigma_{\beta_{k}}^{2},
\end{gathered}
$$

where $\alpha_{k}(\omega)$ and $\beta_{k}(\omega)$ are real-valued random variables. Let $\delta, \alpha, \beta$ be arbitrary numbers such that $0 \leq \alpha<\beta \leq 2 \pi, 0<\delta \leq 1$. $B$ and $C$ are the following subsets

Received by the editors November 11, 2000 and, in revised form, March 23, 2001.

2000 Mathematics Subject Classification. Primary 60H25, 47B80, 34F05.

Key words and phrases. Random polynomials. 
of the complex plane:

$$
\begin{aligned}
& B=\{z: \alpha<\arg z<\beta\}, \\
& C=\{z: 1-\delta \leq|z| \leq 1+\delta\} .
\end{aligned}
$$

$N_{n}(C, \omega)$ and $N_{n}(B, \omega)$, the number of zeros of $F(z, \omega)$ contained in $C$ and $B$, respectively, are integer-valued random variables defined on the infinite-dimensional probability space $\left\langle E^{\infty}, \mathcal{B}^{\infty}, P^{\infty}\right\rangle$, where $E=\mathbb{R}$ or $\mathbb{Z}$ and the probability measure is established by the sequence of independent random variables $\left\{a_{k}(\omega)\right\}_{0}^{\infty}$. (Such a probability space is described, for example, in [3]).

One of the basic limit theorems in the theory of random polynomials was proved by Sparo and Šur [10] (cf. [5]). It states that when the following conditions are satisfied for all polynomials $F_{n}(z, \omega)$ :

(a) the coefficients $\left\{a_{k}(\omega)\right\}_{0}^{n}$ are equidistributed;

(b) $P\left\{a_{k}(\omega)=0, k=0,1, \ldots, n\right\} \neq 1$;

(c) $E\left\{\log ^{+}\left|a_{k}(\omega)\right|\right\}<\infty, k=0,1, \ldots, n$, where $\log ^{+}\left|a_{k}\right|=\max \left\{0, \log \left|a_{k}\right|\right\}$, then the following convergence takes place:

$$
\begin{gathered}
\lim _{n \rightarrow \infty} n^{-1} N_{n}(C, \omega)=1, \\
\lim _{n \rightarrow \infty} n^{-1} N_{n}(B, \omega)=(\beta-\alpha) / 2 \pi
\end{gathered}
$$

in probability.

Arnold [2] improved this theorem and proved that the convergence result (1) holds almost surely and in the $r$-th mean if the moduli of $a_{k}(\omega)$ are equidistributed and $E\left\{\log \left|a_{k}(\omega)\right|\right\}<\infty, k=0,1,2, \ldots$. We shall show here that a more general theorem can be proved and that, in fact, the condition on equidistribution can be dropped. The convergence relations (1) and (2) hold almost surely if all coefficients have finite first and second moments which satisfy certain conditions. This fact can be interpreted in the following way: if the coefficients $a_{k}(\omega), k=0,1,2, \ldots$, satisfy rather general conditions, then the distributions of the zeros of random polynomials $F_{n}(z, \omega)$ tend, in a certain sense, to the uniform distribution on the circumference of the unit circle when $n$ tends to infinity.

In this article, we consider polynomials such that $P\left\{a_{0}(\omega)=0\right\}=0$. We now formulate Theorem 1.

Theorem 1. Let $\left\{a_{k}(\omega)\right\}_{0}^{\infty}$ be a sequence of independent random variables which have continuous densities $f_{k}$ which are uniformly bounded in some neighborhood of the origin with finite means $\mu_{k}$ and standard deviations $\sigma_{k}$ that satisfy the condition

$$
\sup \left\{\varlimsup_{k \rightarrow \infty} \sqrt[k]{\left|\mu_{k}\right|}, \varlimsup_{k \rightarrow \infty} \sqrt[k]{\sigma_{k}}\right\}=1 .
$$

Then (1) and (2) hold almost surely.

We also have the following theorem:

Theorem 2. Let $\left\{a_{k}(\omega)\right\}_{0}^{\infty}$ be a sequence of independent random variables with finite means and standard deviations such that

$$
\varlimsup_{k \rightarrow \infty} \sqrt[k]{\left|\mu_{k}\right|}>\varlimsup_{k \rightarrow \infty} \sqrt[k]{\sigma_{k}}
$$


Then the following equalities hold almost surely:

$$
\begin{aligned}
& \lim _{n_{i} \rightarrow \infty} n_{i}^{-1} N_{n_{i}}\left(C^{*}, \omega\right)=1, \\
& \lim _{n_{i} \rightarrow \infty} n_{i}^{-1} N_{n_{i}}(B, \omega)=(\beta-\alpha) / 2 \pi,
\end{aligned}
$$

where $C^{*}=\left\{z: \frac{1}{\overline{\lim }_{k \rightarrow \infty} \sqrt[k]{\left|\mu_{k}\right|}}-\delta<|z|<\frac{1}{\overline{\lim }_{k \rightarrow \infty} \sqrt[k]{\left|\mu_{k}\right|}}+\delta\right\}$, and $\left\{F_{n_{i}}(z, \omega)\right\}_{0}^{\infty}$ is a subsequence of $\left\{F_{n}(z, \omega)\right\}_{0}^{\infty}$ which consists of polynomials whose coefficients satisfy

$$
\lim _{n_{i} \rightarrow \infty} \sqrt[n_{i}]{\left|\mu_{n_{i}}\right|}=\varlimsup_{k \rightarrow \infty} \sqrt[k]{\left|\mu_{k}\right|}
$$

The interpretation of the theorem is clear: if the radius of convergence of the power series $\sum_{0}^{\infty} \mu_{k} z^{k}$ formed by the means is strictly less than the radius of converence of the power series $\sum_{0}^{\infty} \sigma_{k} z^{k}$ formed by the standard deviations, then almost surely a subsequence of $\left\{F_{n}(z, \omega)\right\}_{0}^{\infty}$ can be found such that for polynomials of sufficiently high order belonging to the subsequence, the distribution of zeros is close to uniform on the circumference of some circle, not necessarily the unit circle, with center at the origin.

Our third and final theorem is a probabilistic analog of the Cauchy-Hadamard theorem on the radius of convergence of a numerical power series. It enables one to find radii of convergence for random power series defined on an infinite-dimensional probability space established by a sequence of independent random variables with finite means and standard deviations. For the specific case where the moduli of the coefficients $a_{k}(\omega)$ not only are independent but are also identically distributed random variables, the problem of determining the radius of convergence has already been investigated by Arnold [1] (cf. the discussion in Bharucha-Reid [4, page 44]).

Theorem 3. (a) For a sequence of independent random variables $\left\{a_{k}(\omega)\right\}_{0}^{\infty}$ with finite means and standard deviations which satisfy condition (4), the radius of convergence of the random power series $\sum_{0}^{\infty} a_{k}(\omega) z^{k}$ almost surely equals $\frac{1}{\lim _{k \rightarrow \infty} \sqrt[k]{\left|\mu_{k}\right|}}$.

(b) If for a sequence of independent random variables $\left\{a_{k}(\omega)\right\}_{0}^{\infty}$ with finite means and standard deviations which satisfy the inequality

$$
\varlimsup_{k \rightarrow \infty} \sqrt[k]{\sigma_{k}} \geq \varlimsup_{k \rightarrow \infty} \sqrt[k]{\left|\mu_{k}\right|}
$$

there exists a subsequence $\left\{a_{k_{j}}(\omega)\right\}_{0}^{\infty}$ such that the conditions

$$
\begin{aligned}
& \sum_{k=0}^{\infty} P\left\{\left|a_{k_{j}}(\omega)\right|>\frac{1}{k_{j}} \sigma_{k_{j}}\right\}=\infty, \\
& \lim _{k_{j} \rightarrow \infty} \sqrt[k]{\sigma_{k_{j}}}=\varlimsup_{k \rightarrow \infty} \sqrt[k]{\sigma_{k}}
\end{aligned}
$$

are satisfied, then the radius of convergence of the random power series $\sum_{k=0}^{\infty} a_{k}(\omega) z^{k}$ almost surely equals $\frac{1}{\lim _{k \rightarrow \infty} \sqrt[k]{\sigma_{k}}}$.

We note that the set of conditions in (6) holds for a power series whose members are distributed according to the Gaussian law, the uniform law, or any one of many other frequently encountered laws. 


\section{§2. Proof of Theorems $1-3$}

The proof of Theorem 1 utilizes Lemmas 1-3.

Lemma 1. If $\left\{a_{k}(\omega)\right\}_{0}^{\infty}$ is a sequence of random variables with finite means and standard deviations, then

$$
\varlimsup_{k \rightarrow \infty} \sqrt[k]{\left|a_{k}\right|} \leq \sup \left\{\varlimsup_{k \rightarrow \infty} \sqrt[k]{\sigma_{k}}, \varlimsup_{k \rightarrow \infty} \sqrt[k]{\left|\mu_{k}\right|}\right\}
$$

almost surely.

Proof. We can express $\left\{a_{k}(\omega)\right\}$ as a sum of two sequences:

$$
\begin{aligned}
& \eta_{k}(\omega)= \begin{cases}a_{k}(\omega), & \text { if }\left|\mu_{k}\right|>\sigma_{k}, \\
0, & \text { otherwise }\end{cases} \\
& \eta_{k}^{\prime}(\omega)= \begin{cases}a_{k}(\omega), & \text { if }\left|\mu_{k}\right| \leq \sigma_{k}, \\
0, & \text { otherwise }\end{cases}
\end{aligned}
$$

The Chebyshev inequality implies that

$$
P\left\{\left|\eta_{k}(\omega)-\mu_{k}\right|>(k-1)\left|\mu_{k}\right|\right\} \leq \frac{\sigma_{k}^{2}}{(k-1)^{2} \mu_{k}^{2}} \leq \frac{1}{(k-1)^{2}} .
$$

From this inequality, it follows that

$$
\sum_{k=1}^{\infty} P\left\{\left|\eta_{k}(\omega)\right|>k\left|\mu_{k}\right|\right\}<\infty
$$

In view of the equality

$$
\varlimsup_{k \rightarrow \infty} \sqrt[k]{\left|\mu_{k}\right|}=\varlimsup_{k \rightarrow \infty} \sqrt[k]{k\left|\mu_{k}\right|}
$$

and the Borel-Cantelli zero-one law, we conclude that

$$
\varlimsup_{k \rightarrow \infty} \sqrt[k]{\left|\eta_{k}(\omega)\right|} \leq \varlimsup_{k \rightarrow \infty} \sqrt[k]{\left|\mu_{k}\right|}
$$

almost surely.

Now we use the Chebyshev inequality for the sequence $\left\{\eta_{k}^{\prime}(\omega)\right\}_{0}^{\infty}$ :

$$
P\left\{\left|\eta_{k}^{\prime}(\omega)-\mu_{k}\right|>k \sigma_{k}\right\} \leq \frac{\sigma_{k}^{2}}{k^{2} \sigma_{k}^{2}}=\frac{1}{k^{2}}
$$

for all $k$ such that $\eta_{k}^{\prime} \not \equiv 0$.

From this inequality, it follows that

$$
\sum_{k=0}^{\infty} P\left\{\left|\mu_{k}^{\prime}\right|>(k+1) \sigma_{k}\right\}<\infty .
$$

We conclude that

$$
\varlimsup_{k \rightarrow \infty} \sqrt[k]{\left|\eta_{k}^{\prime}(\omega)\right|} \leq \varlimsup_{k \rightarrow \infty} \sqrt[k]{\sigma_{k}}
$$

almost surely. Since

$$
\varlimsup_{k \rightarrow \infty} \sqrt[k]{\left|a_{k}\right|} \leq \sup \left\{\varlimsup_{k \rightarrow \infty} \sqrt[k]{\left|\eta_{k}\right|}, \varlimsup_{k \rightarrow \infty} \sqrt[k]{\left|\eta_{k}^{\prime}\right|}\right\},
$$

the lemma is proved. 
Lemma 2. If for a sequence of independent random variables $\left\{a_{k}(\omega)\right\}_{0}^{\infty}$ with continuous densities $f_{k}$, a number $A$ can be found such that in some $\varepsilon$-neighborhood of the origin $\{z:|z|<\varepsilon\}$ the inequality $\left|f_{k}(z)\right|<A$ is satisfied for any $k$, then almost surely

$$
\varliminf_{k \rightarrow \infty} \sqrt[k]{\left|a_{k}\right|}=1
$$

Proof. Let $b$ be some fixed number that belongs to the interval $(0,1)$. It is clear that if the conditions of the lemma are satisfied for any $k$, then the inequality

$$
P\left\{\left|a_{k}(\omega)\right|<b^{k}\right\}<\pi A b^{2 k}
$$

is satisfied for any $k \geq N$, where $N$ is any integer such that $b^{N}<\varepsilon$. From (14) we get

$$
\sum_{k=0}^{\infty} P\left\{\left|a_{k}(\omega)\right|<b^{k}\right\}<\infty,
$$

which, in turn, implies that

$$
{\underline{\varliminf_{k \rightarrow \infty}}}_{k \rightarrow \infty} \sqrt[k]{\left|a_{k}(\omega)\right|} \geq b
$$

almost surely. This proves the lemma, since $b$ was chosen arbitrarily.

Lemma 3 is due to Erdös and Turan [8]:

Lemma 3. Let $a_{k}, k=0,1, \ldots, n$, be arbitrary complex numbers not all of which are equal to zero, and let $N(\alpha, \beta)$ denote the number of zeros of $F(z)=\sum_{0}^{n} a_{k} z^{k}$ which belong to the sector $0 \leq \alpha \arg z<\beta$. Then, for $a_{0} a_{n} \neq 0$,

$$
\left|N(\alpha, \beta)-\frac{(\beta-\alpha) n}{2 \pi}\right|<16\left[n \log \frac{\sum_{0}^{n}\left|a_{k}\right|}{\left(\left|a_{0} a_{n}\right|\right)^{1 / 2}}\right]^{1 / 2} .
$$

Remark. This result of Erdös and Turan was also utilized by Sparo and Šur in proving their result that we quoted earlier in Section 1.

We note that Lemmas 1 and 2 imply that in the infinite-dimensional probability space defined by a sequence $\left\{a_{k}(\omega)\right\}_{0}^{\infty}$ which satisfies the conditions of Theorem 1 , the measure of the set of sequences

$$
E=\left\{\left\{a_{k}\right\}_{0}^{\infty}: \lim _{k \rightarrow \infty} \sqrt[k]{\left|a_{k}\right|}=1\right\}
$$

equals one.

Now let us consider an arbitrary number sequence $\left\{a_{k}\right\}_{1}^{\infty}$ which belongs to $E$, and the corresponding sequence of polynomials $\left\{F_{n}(z)=\sum_{0}^{n} a_{k} z^{k}\right\}_{0}^{\infty}$. Let $K_{1}^{n}(a)$ denote the number of zeros of $F_{n}(z)$ belonging to the domain $\{z:|z|<1-a\}$, $K_{2}^{n}(a, b)$ the number of zeros of $F_{n}(z)$ belonging to the domain $\{z: 1-a \leq$ $|z| \leq 1+b\}$, and $K_{3}^{n}(b)$ the number of zeros of $F_{n}(z)$ belonging to the domain $\{z:|a|>1+b\}$. Let $G>0$ be a lower bound of the set of absolute values of zeros 
of all polynomials $F_{n}(z), n=0,1,2, \ldots$. The Hurwitz inequality implies that for any $\delta \in(0,1)$,

$$
\lim _{n \rightarrow \infty} n^{-1} K_{1}^{n}(\delta)=0 .
$$

Therefore, in order to prove the first assertion of Theorem 1, it is enough to show that for any $\delta \in(0,1)$, we have

$$
\lim _{n \rightarrow \infty} n^{-1} K_{3}^{n}(\delta)=0 .
$$

Assume that the last assertion is not true. Then for any numbers $\delta$ and $c$ and for any integer $L$, there exists $\ell>L$ such that

$$
\frac{K_{3}^{\ell}(\delta)}{\ell}>c
$$

We stress that in our considerations below, $\delta$ and $c$ will be fixed numbers. We find a number $\delta^{\prime} \in(0,1)$ which satisfies the following inequality:

$$
\left(\frac{1}{1-\delta^{\prime}}\right)^{1-c}<(1+\delta)^{\frac{c}{4}}
$$

Assume $L=\max \left\{N^{\prime}, N^{\prime \prime}, N^{\prime \prime \prime}\right\}$, where $N^{\prime}$ is an integer such that for any $n \geq N^{\prime}$ we have

$$
(1+\delta)^{\frac{n c}{4}}>\frac{1}{\left|a_{n}\right|}
$$

$N^{\prime \prime}$ is an integer such that for any $n \geq N^{\prime \prime}$ we have

$$
(1+\delta)^{\frac{n c}{4}}>\left|a_{0}\right|
$$

and $N^{\prime \prime \prime}$ is an integer such that for any $n \geq N^{\prime \prime \prime}$ we have

$$
(1+\delta)^{\frac{n c}{4}}>\frac{1}{G^{K_{1}^{n}\left(\delta^{\prime}\right)}} .
$$

Then, for any $n \geq L$, the following inequality is satisfied:

$$
(1+\delta)^{n c}>\left(\frac{1}{1-\delta^{\prime}}\right)^{(1-c) n} \cdot \frac{1}{\left|a_{n}\right|} \cdot\left|a_{0}\right| \cdot \frac{1}{G^{K_{1}^{n}\left(\delta^{\prime}\right)}} .
$$

Inequality (21) is equivalent to

$$
(1+\delta)^{c n} \cdot G^{K_{1}^{n}\left(\delta^{\prime}\right)} \cdot\left(1-\delta^{\prime}\right)^{(1-c) n}>\frac{\left|a_{0}\right|}{\left|a_{n}\right|} .
$$

Let $\ell \geq L$ be an integer such that $K_{3}^{\ell}(\delta)>\ell c$. Then the product of the moduli of the zeros of $F_{\ell}(z)$ which belong to $\{z:|z|>1+\delta\}$ is greater than $(1+\delta)^{c \ell}$, the product of the moduli of the zeros of $F_{\ell}(z)$ belonging to $\left\{z: 1-\delta^{\prime}>|z|\right\}$ is greater than $G^{K_{1}^{\ell}\left(\delta^{\prime}\right)}$, and the product of the moduli of the zeros of $F_{\ell}(z)$ which belong to $\left\{z: 1-\delta^{\prime}<|z|<1+\delta\right\}$ is greater than $\left(1-\delta^{\prime}\right)^{(1-c) \ell}$. In view of (22), the product of the moduli of all zeros of $F_{\ell}(z)$ turns out to be greater than $\left|\frac{a_{0}}{a_{\ell}}\right|$, which is impossible. This contradiction proves that for any number sequences $\left\{a_{k}\right\}_{0}^{\infty}$ belonging to $E$, the relative number of zeros of $F_{n}(z)$ belonging to $C$ tends to one as $n$ tends to infinity for any $\delta$.

Thus the first assertion of Theorem 1 is proved. 
In view of Lemma 3 , in order to prove the second assertion of Theorem 1 , it is enough to utilize the fact that for any sequence $\left\{a_{k}\right\}_{0}^{\infty}$ belonging to $E$, all elements of which are nonzero, we have

$$
\lim _{n \rightarrow \infty} \frac{1}{n} \log \frac{\sum_{0}^{n}\left|a_{k}\right|}{\left|a_{0} a_{n}\right|^{1 / 2}}=0
$$

In order to prove Theorem 2, we first prove Lemma 4.

Lemma 4. If for a sequence of independent random variables $\left\{a_{k}(\omega)\right\}_{0}^{\infty}$ with finite means and standard deviations, the inequality

$$
\varlimsup_{k \rightarrow \infty} \sqrt[k]{\left|\mu_{k}\right|}>\varlimsup_{k \rightarrow \infty} \sqrt[k]{\sigma_{k}}
$$

is satisfied, then for any subsequence $\left\{a_{k_{i}}(\omega)\right\}_{0}^{\infty}$ such that

$$
\lim _{k_{i} \rightarrow \infty} \sqrt[k_{i}]{\left|\mu_{k_{i}}\right|}=\varlimsup_{k \rightarrow \infty} \sqrt[k]{\left|\mu_{k}\right|}
$$

we have that

$$
\lim _{k_{i} \rightarrow \infty} \sqrt[k_{i}]{\left|a_{k_{i}}\right|}=\varlimsup_{k \rightarrow \infty} \sqrt[k]{\left|\mu_{k}\right|}
$$

almost surely.

Proof. We note that for arbitrary $\delta>0$, there is an integer $N_{1}$ such that for any $k_{i} \geq N_{1}, \sqrt[k_{i}]{\left|\mu_{k_{i}}\right|}>R_{1}-\delta$, where $R_{1}=\bar{\varlimsup}_{k \rightarrow \infty} \sqrt[k]{\left|\mu_{k}\right|}$. Since $\lim _{k_{i} \rightarrow \infty} \sqrt{\sigma_{k_{i}}} \leq$ $R_{2}=\varlimsup_{k \rightarrow \infty} \sqrt{\sigma_{k}}$ for any $\delta>0$, there is an integer $N_{2}$ such that for any $k_{i} \geq N_{2}$, $\sqrt[k_{i}]{\sigma_{k_{i}}}<R_{2}+\delta$. Let us choose $\delta$ so that

$$
\frac{R_{2}+\delta}{R_{1}-\delta}=p<1
$$

Then for any $k_{i} \geq \sup \left\{N_{1}, N_{2}\right\}$, the following inequality is satisfied:

$$
\frac{\sigma_{k_{i}}^{2}}{\mu_{k_{i}}^{2}}<p^{2 k_{i}}
$$

This inequality, together with the inequality

$$
P\left\{\left|\mu_{k_{i}}-a_{k_{i}}(\omega)\right|>\frac{1}{2}\left|\mu_{k_{i}}\right|\right\}<\frac{4 \delta_{k_{i}}^{2}}{\mu_{k_{i}}^{2}},
$$

implies that

$$
\sum_{i=1}^{\infty} P\left\{\left|\mu_{k_{i}}-a_{k_{i}}(\omega)\right|>\frac{1}{2}\left|\mu_{k_{i}}\right|\right\}<\infty .
$$

This proves the lemma.

Lemma 4 implies that in a probability space defined by a sequence $\left\{a_{k}(\omega)\right\}_{0}^{\infty}$ which satisfies the conditions of Theorem 2, the measure of the set

$$
E^{*}=\left\{\left\{a_{k}\right\}_{0}^{\infty}: \varlimsup_{k \rightarrow \infty} \sqrt[k]{\left|a_{k}\right|}=\lim _{k_{i} \rightarrow \infty} \sqrt[k_{i}]{\left|a_{k_{i}}\right|}=\varlimsup_{k \rightarrow \infty} \sqrt[k]{\left|\mu_{k}\right|}\right\}
$$

equals one. 
Consider an arbitrarily chosen sequence $\left\{a_{k}\right\}_{0}^{\infty}$ belonging to $E^{*}$. Obviously, for the sequence

$$
\left\{c_{k}\right\}_{0}^{\infty}=\left\{\frac{a_{k}}{\left(\varlimsup_{k \rightarrow \infty} \sqrt[k]{\left|\mu_{k}\right|}\right)^{k}}\right\}_{0}^{\infty}
$$

we have

$$
\varlimsup_{k \rightarrow \infty} \sqrt[k]{\left|c_{k}\right|}=\lim _{k_{i} \rightarrow \infty} \sqrt[k_{i}]{\left|c_{k_{i}}\right|}=1 .
$$

In a way similar to the technique used in the proof of Theorem 1 , we can show that for arbitrary $\delta>0, \alpha, \beta$ such that $0 \leq \alpha<\beta<2 \pi$, we have the convegence results

$$
\begin{gathered}
\lim _{n_{i} \rightarrow \infty} n_{i}^{-1} N_{n_{i}}(B)=\frac{\beta-\alpha}{2 \pi}, \\
\lim _{n_{i} \rightarrow \infty} n_{i}^{-1} N_{n_{i}}\left(C^{\prime}\right)=1,
\end{gathered}
$$

where $C^{\prime}=\left\{1-\delta \varlimsup_{k \rightarrow \infty} \sqrt[k]{\left|\mu_{k}\right|}<|z|<1+\delta \varlimsup_{k \rightarrow \infty} \sqrt[k]{\left|\mu_{k}\right|}\right\}$, and $N_{n_{i}}(B)$ and $N_{n_{i}}\left(C^{\prime}\right)$ are the number of zeros of $\sum_{0}^{n_{i}} c_{k} z^{k}$ belonging to $B$ and $C^{\prime}$, respectively.

Since every zero of the polynomial $\sum_{0}^{n_{i}} a_{k} z^{k}$ can be expressed as a corresponding zero of the polynomial $\sum_{0}^{n_{i}} c_{k} z^{k}$ divided by $\varlimsup_{k \rightarrow \infty} \sqrt[k]{\left|\mu_{k}\right|}$, Theorem 2 is proved.

Corollary. If, in addition to the conditions of Theorem 2, a sequence of random variables $\left\{a_{k}(\omega)\right\}_{0}^{\infty}$ satisfies the condition

$$
\varlimsup_{k \rightarrow \infty} \sqrt[k]{\left|\mu_{k}\right|}=\lim _{k \rightarrow \infty} \sqrt[k]{\left|\mu_{k}\right|}
$$

then for arbitrary $\delta>0, \alpha, \beta$ such that $0 \leq \alpha<\beta<2 \pi$, we have

$$
\begin{aligned}
& \lim _{n \rightarrow \infty} n^{-1} N_{n}\left(C^{*}, \omega\right)=1, \\
& \lim _{n \rightarrow \infty} n^{-1} N_{n}(B, \omega)=\frac{\beta-\alpha}{2 \pi},
\end{aligned}
$$

almost surely.

Proof of Theorem 3. The first assertion of the theorem follows directly from Lemmas 1 and 4. We now prove the second assertion of the theorem. From conditions (6), it follows that

$$
\varlimsup_{k \rightarrow \infty} \sqrt[k]{\left|a_{k}\right|} \geq \varlimsup_{k \rightarrow \infty} \sqrt[k]{\sigma_{k}}
$$

almost surely. Lemma 1 implies that $\varlimsup_{k \rightarrow \infty} \sqrt[k]{\left|a_{k}\right|} \leq \varlimsup_{k \rightarrow \infty} \sqrt[k]{\delta_{k}}$ almost surely provided that $\varlimsup_{k \rightarrow \infty} \sqrt[k]{\sigma_{k}} \geq \varlimsup_{k \rightarrow \infty} \sqrt[k]{\left|\mu_{k}\right|}$. This completes the proof. 


\section{§3. Estimation of the Degree of CONVERGENCE}

Theorem 1 on the asymptotic behavior of zeros of random polynomials does not enable us to evaluate the degree of convergence of the sequence of random variables

$$
\left\{\zeta_{n}\right\}_{n=0}^{\infty}=\left\{\frac{N_{n}(B, \omega)}{n}\right\}_{0}^{\infty}, \quad B=\{z: \alpha<\arg z<\beta\},
$$

defined on the infinite-dimensional probability space $\left\langle E^{\infty}, B^{\infty}, P^{\infty}\right\rangle$ to the constant $\frac{\beta-\alpha}{2 \pi}$ if the sequence $\left\{a_{k}(\omega)\right\}_{0}^{\infty}$ does not satisfy some additional conditions. For the case where means and standard deviations of the coefficients are uniformly bounded, the degree of convergence can be estimated. This enables us to estimate numerically the joint distribution of zeros for polynomials of high degrees. We now show how this can be done.

Let $p$ and $\varepsilon$ be fixed numbers, let $E^{*}$ denote $\sup \left\{\mu\left|a_{n}(\omega)\right|\right\}_{0}^{\infty}$, and let $\sigma^{*}$ denote $\sup \left\{\sigma\left|a_{n}(\omega)\right|\right\}_{0}^{\infty}$. Then, there is an integer $N$ such that for any $n \geq N$,

$$
P\left\{\left|\zeta_{n}-\frac{\beta-\alpha}{2 \pi}\right|<\varepsilon\right\}>1-p
$$

We now give a procedure to determine the value $N$. From the Chebyshev inequality, it follows that

$$
P\left\{\left|\sum_{0}^{n}\right| a_{k}(\omega)\left|-\mu\left(\sum_{0}^{n}\left|a_{k}(\omega)\right|\right)\right| \geq \sqrt{n+1} \sigma\left(\sum_{0}^{n}\left|a_{k}(\omega)\right|\right)\right\} \leq \frac{1}{n+1} .
$$

Since $\left\{a_{k}(\omega)\right\}_{0}^{n}$ are independent, the following inequalities are satisfied:

$$
\begin{aligned}
& E\left(\sum_{0}^{n}\left|a_{k}(\omega)\right|\right) \leq(n+1) E^{*} \\
& \sigma\left(\sum_{0}^{n}\left|a_{k}(\omega)\right|\right) \leq \sqrt{n+1} \sigma^{*} .
\end{aligned}
$$

Inequalities (29)-(31) imply that

$$
P\left\{\sum_{0}^{n}\left|a_{k}(\omega)\right| \geq(n+1)\left(E^{*}+\sigma^{*}\right)\right\} \leq \frac{1}{n+1} .
$$

Let $A$ be a number such that for any $n \geq 0$,

$$
P\left\{\frac{1}{\left|a_{n}(\omega)\right|}>A\right\}<\frac{p}{4}
$$

In order to find $A$, we can utilize the uniform boundedness of the densities $f_{k}$ of $\left\{a_{k}(\omega)\right\}_{0}^{\infty}$ in some neighborhood of the origin. Let $N^{\prime}$ be an integer such that for any $n \geq N^{\prime}$,

$$
\frac{1}{n+1}<\frac{p}{2}
$$

From (32)-(34), it follows that for any $n \geq N^{\prime}$,

$$
P\left\{\frac{\sum_{0}^{n}\left|a_{k}(\omega)\right|}{\left|a_{0}(\omega) a_{n}(\omega)\right|^{1 / 2}}>(n+1)\left(E^{*}+\sigma^{*}\right) A\right\}<p .
$$


Let $N^{\prime \prime}$ be an integer such that for any $n \geq N^{\prime \prime}$, the following inequality is satisfied:

$$
16\left[\frac{\log \left[(n+1)\left(E^{*}+\sigma^{*}\right) A\right]}{n}\right]<\varepsilon .
$$

Let $N$ denote $\max \left(N^{\prime}, N^{\prime \prime}\right)$. Then, by Lemma 3 , for any $n \geq N$, the relation (28) is satisfied.

\section{REFERENCES}

1. Arnold, L., Über die Konvergenz einer zufälligen Potenzreihe, J. Reine Angew. Math. 222 (1966), 79-112. MR 32:6521

2. Arnold, L., Über die Nullstellenverteibung züfalliger Polynome, Math. Z. 92 (1966), 12-18. MR 34:851

3. Bauer, H., Probability Theory and Elements of Measure Theory, Academic Press, New York, 1978, pp. 162-164. MR 80b:6001

4. Bharucha-Reid, A.T., Probabilistic Methods in Applied Mathematics, vol. 2, Academic Press, New York, 1970. MR 41:4701b

5. Bharucha-Reid, A.T. and Sambandham, M., Random Polynomials, Academic Press, New York, 1986. MR 87m:60118

6. Bogomolny, E., Bohigas, O. and Leboeuf, P., Quantum chaotic dynamics and random polynomials, J. Stat. Phys. 85 (1996), 639-679. MR 98a:81046

7. Edelman, A. and Kostlan, E., How many zeros of a random polynomial are real?, Bulletin (New Series) of the American Math. Soc. 32 (1995), 1-37. MR 95m:60082

8. Erdos, P. and Turan, P., On the distribution of roots of polynomials, Ann. Math. 51 (1950), 105-119. MR 11:431b

9. Shmerling, E., Linear Systems with Random Coefficients, Ph.D. dissertation, Bar-Ilan University, Israel, 2000.

10. Sparo, D.I. and Šur, M.G., On the distribution of roots of random polynomials, Vestn. Mosk. Univ. Ser. 1 Mat. Mekh. (1962), 40-43. MR 25:2635

Department of Mathematics, College of Judea and Samaria, 44837 Ariel, Israel

Department of Mathematics and Computer Science, Bar-Ilan University, 52900 RAMAT-Gan, IsRael

E-mail address: hochberg@macs.biu.ac.il 